dels. Nirgends aber gibt es eine Angabe über die zur Kohlenstoffbestimmung angewandte Methode. Nach der oben angegebenen Methode wurden folgende Nickelsorten untersucht:

1. Reinstes Würfelnickel von der Nickelaktiengesellschaft Fleitmann \& Witte, Iserlohn:

$\begin{array}{cccc}\text { I. } & \text { II. } & \text { Si } \\ \text { Gelöst: } & 4,2624 \mathrm{~g} & 4,9788 \mathrm{~g} & 4,9788 \mathrm{~g} \\ \mathrm{CO}_{2}: 0,0784 \mathrm{~g} & 0,0946 \mathrm{~g} & \mathrm{SiO}_{2}: 0,0160 \mathrm{~g} \\ \mathrm{C}: 0,50 \% & 0,52 \% & \mathrm{Si}: 0,15 \% & 0.15 \%\end{array}$
delphia:

2. Amerikanisches Rohnickel aus Phila$\begin{array}{rcccc} & \text { I. } & \text { II. } & \text { Si } \\ \text { Gelöst: } & 5,8866 \mathrm{~g} & 6,5480 \mathrm{~g} & 5,8866 \mathrm{~g} \\ \text { CO. }: 0,2717 \mathrm{~g} & 0,3233 \mathrm{~g} & \mathrm{SiO}_{2}: 0,1060 \mathrm{~g} \\ \mathrm{C}: 1,26 \% & 1,34 \% & \text { Si: } 0,84 \% .\end{array}$

Die Versuche zur Darstellung von Nickelkohlenstofflegierungen erstreckten sich wiederum zuerst auf die Kohlenstoffaufnahme des Metalls bei seiner Schmelztemperatur oder wenig höher und dann bei der Hitze des elektrischen Flammenbogens. Als Ausgangsmaterial diente das oben untersuchte reinste Würfelnickel, welches durch Lösen in Salpetersäure, Glühen und Reduzieren im Wasserstoffstrome in Metallpulver übergeführt wurde.

Versuch 1. 10 g Nickel mit 0,5 g Ruß gemischt gaben nach zweistündigem Erhitzen im Koksofen eine große Anzahl kleiner Metallkugeln. Nach dem Abblasen des überschüssigen Kohlenstoffs konnte aus diesen durch Zusammenschmelzen ein größeres Metallstück erhalten werden. Die Farbe der Bruchfläche war graphitähnlich und röllig gleichmäßig. Das Metall war so weich und zähe, $d a B$ es mit dem Meißel ähnlich wie Blei zerschnitten werden konnte. Vielleicht dürfte das so erhaltene Metall dem von Ross und Irving mit Nickelgraphit bezeichneten Produkte entsprechen. Die Analyse wurde im Druckrohr vorgenommen.

$$
\begin{gathered}
\text { Gelist: } 2,5986 \mathrm{~g} \\
\mathrm{CO}_{2}: 0,1630 \mathrm{~g} \\
\mathrm{C}: 1,71 \%
\end{gathered}
$$

Versuch 2. $10 \mathrm{~g}$ Nickel mit $0,8 \mathrm{~g}$ RuB 3 Stunden lang im Koksofen mit Gebläse auf höchste WeiGglut erhitzt, lieferten wiederum zahlreiche kleine Metallkugeln, welche für sich zusammengeschmolzen eine Metallkugel gaben, die dem in Versuch 1 erhaltenen Produkte äußerlich völlig gleich sah.

Analyse. Gelöst: 4,7228.g

$$
\begin{gathered}
\mathrm{CO}_{2}: 0,3710 \mathrm{~g} \\
\mathrm{C}: 2,14 \%
\end{gathered}
$$

Ein bemerkenswerter Unterschied zeigte sich zwischen den bis jetzt erhaltenen Kohlenstoffnickellegierungen und den Kohlenstoff- kobaltlegierungen von annähernd demselben Kohlenstoffgehalt. Während bei Kobalt ein äußerst hartes Metall mit kristallinischer Struktur entstand, sind die Nickellegierungen sehr weich, ohne Kristallstruktur, ähnlich dem durch elektrische Schmelzung erhaltenen Kohlenstoffkobalt, und unterscheiden sich äußerlich nicht von dem in den folgenden Versuchen durch elektrische Schmelzung erhaltenen Kohlenstoffnickel.

Versuch 3. $20 \mathrm{~g}$ Nickel wurden mit 2,5 g Rub zusammengeprebt und 10 Minuten lang im Flammenbogen erhitzt. Die erhaltene Metallkugel spaltete beim Zerschlagen in dünne Blätter, doch war es auch mit bewaffnetem Auge unmöglich, den graphitischen Kohlenstoff als solchen von dem Metalle zu unterscheiden.

Die Analyse im Druckrohr ergab:

$$
\begin{gathered}
\text { Gelöst: } 0,8310 \mathrm{~g} \\
\mathrm{CO}_{\mathrm{g}}: 0,1400 \mathrm{~g} \\
\mathrm{C}: 4,69 \%
\end{gathered}
$$

Versuch 4. $20 \mathrm{~g}$ Nickel mit $4 \mathrm{~g}$ RuB gemischt ließen sich nur zu kleinen Metalltropfen schmelzen. Erst durch wiederholtes Abblasen des Kohlenstoffs und Schmelzen konnte hieraus ein größeres Metallkorn erhalten werden.

$$
\begin{aligned}
& \text { Analyse. Gelöst: } 0,6120 \mathrm{~g} \\
& \mathrm{CO}_{2}: 0,1404 \mathrm{~g} \\
& \text { C: } 6,25 \%
\end{aligned}
$$

Aus den Versuchen geht hervor, daß das Nickel ebenfalls große Neigung besitzt, im geschmolzenen Zustande Kohlenstoff aufzunehmen. Während aber bei Eisen und Kobalt ein großer Teil des Kohlenstoffs in dem erkalteten Metalle im chemisch verbundenen Zustande verbleibt, ist der von Nickel gelöste Kohlenstoff fast nur als graphitischer Kohlenstoff vorhanden. Als die höchste Kohlungsstufe des Nickels muß die Legierung mit etwa $6,25 \%$ Kohlenstoff angesehen werden.

(Schlus folgt.)

\section{Bestimmung von Kohlendioxyd neben Chlor besonders im elektrolytischen Chlor.}

\section{$d$ Ton Dr. Ing. Mix Schlötrer. (Eingeg. d. 8.1. 1904.)}

Unter diesem Titel gibt C. Offerhaus') einen Auszug aus seiner Dissertation und führt darin zur Bestimmung dieser in einem Gemisch nebeneinander vorhandenen Gase drei Methoden an, die im wesentlichen auf Absorption des Chlors und des Kohlendioxyds in Natriumhydroxydlösung beruhen. Dabei

1) Diese Z. 16, 1033, (1903). 
wird entweder das Chlor ermittelt durch Titration des aus Kaliumjodidlösung freigemachten Jods oder die Kohlensäure durch Titration mit Salzsäure oder Oxalsäure. Dadurch, daß titrimetrische und gasometrische Bestimmung nebeneinander hergehen, also Volumen und Gewicht zueinander in Beziehung treten, wird eine langwierige Umrechnung der Resultate bedingt, die sich freilich durch Tabellen oder Faktoren vereinfachen liebe. Kurz mir scheint, dab die von Offerhaus angegebenen Methoden der für den praktischen Chemiker notwendigen Einfachheit ermangeln.

Man sollte meinen, gerarle ein so reaktionsfähiges Element, wie das Chlor, böte Angriffspunkte genug, die sich zu einer gasometrischen Bestimmung desselben benutzen ließen. Tatsächlich hat ja auch Winkler die Fähigkeit des Chlors, Ferroverbindung zu Ferriverbindung zu oxydieren, zu einer gasometrischen Bestimmung desselben benutzt. Möglicherweise liebe sich auch die salzsaure Kuprochloridlösung, wie sie zur gasometrischen Bestimmung des Kohlenoxyds dient, zur Absorption des Chlors benutzen.

Ich bediente mich zur Absorption des Chlors in Gasgemischen des Hydrazins, bezw. seiner Salze.

Wie ich an anderer Stelle ${ }^{2}$ ) ausgeführt habe, wirken die Halogene auf Hydrazinsulfat unter Entwicklung von Stickstoff im Sinne der Gleichung:

$$
\mathrm{N}_{2} \mathrm{H}_{4}+2 \mathrm{Cl}_{2}=4 \mathrm{HCl}+\mathrm{N}_{2}
$$

d. h. je zwei Volumina Chlor entsprechen einem Volumen Stickstoff.

Die Ausführung der Analyse gestaltet sich einfach. Man mißt in die Bunte-Bürette $100 \mathrm{ccm}$ des zu untersuchenden Gases. Sollen dieVolumenprozente später in Gewichtsprozente umgerechnet werden, darf man beim Einstellen das Niveaugefäß der Bunte-Bürette nur mit wenig Wasser als Absperrflüssigkeit füllen, doch tritt auch bei der üblichen Arbeitsweise mit 20 oder $25 \mathrm{ccm}$ Wasser kein merkbarer Fehler auf.

Hat man $100 \mathrm{ccm}$ abgemessen, so saugt man Wasser aus der Bürette und läßt dafür

2) Z. anorg. Chem. 37, 164 u. ff.
Hydrazinsulfatlösung eintreten. Nachdem man einige Minuten umgeschüttelt hat, läßt man ein zweites Mal Hydrazinsulfatlösung einsaugen, was leicht geschieht, da fiir zwei Volumina Chlor nur ein Volumen Stickstoff entwickelt wird. Ist die Reaktion zu Ende, wovon man sich dadurch ïberzeugen kamn, daß das Gasgemisch an Volumen nicht mehr abnimmt -- dies geschieht, indem man von oben Wrasser bis zum Ausgleich des Druckes zulaufen läßt —, so liest man nach einigem Warten die Volumenabnalıme ab. Verdoppelt man diese Volumenverminderung, so hat man direkt die Volumenprozente Chlor.

Vor Bestimmung der Kohlensäure ist es zweckmäßig, die Hydrazinlösung so weit wie möglich abzusaugen, da nach R. Stolle ${ }^{3}$ ) Hydrazinlösungen bei Gegenwart von Carbonaten oder Bicarbonaten sich leicht zersetzen.

Ich habe diese Bestimmungsmethode bei Gasgemischen angewandt, in denen Chlor bis zu einigen Prozenten enthalten war.

Um sie auf ihre Brauchbarkeit auch bei höherem Chlorgehalt zu untersuchen, stellte ich mir, wie Offerhaus, Gemische von Kohlensäure, Chlor und Luft her und bestimmte das eine Mal in demselben den Gehalt an Chlor und Kohlensäure nach Offerhaus, nämlich durch Absorption beider mit 1/5-n.-Natriumhydroxydlösung und Bestimmung des Chlors durch das ans Kaliumjodid ausgeschiedene Jod, das andere Mal mit $\mathrm{Hydr}_{\mathrm{r}}$ azinsulfat. Ich lasse hier die erhaltenen Resultate folgen:

nach offerbaus

Volumenprozente mit Hydrazinsulfat 1.

$42,86 \% \mathrm{Cl} \quad 9,5 \% \mathrm{CO}_{2}, 42,8 \% \mathrm{Cl} \quad 9,6 \% \mathrm{CO}_{2}$ 2.

$23,6 \%$ Cl $58,2 \% \mathrm{CO}, 24,0 \% \mathrm{Cl} 58,0 \% \mathrm{CO}_{2}$ 3.

$33,8 \% \mathrm{Cl} 13,0 \% \mathrm{CO}, 33,6 \% \mathrm{Cl} 13,2 \% \mathrm{CO}_{2}$. Aus diesen Werten ist zu ersehen, daß die Hydrazinmethode bei Gasgemischen von verschiedenstem Chlorgehalte zuverlässige Werte gibt. Sie hat vor den von Offerhaus angegebenen Methoden den Vorzug der Einfachheit und rascheren Ausführbarkeit voraus.

Bernburg, den 1. Januar 1904.

3) J. prakt. Chem. 66, 332, (1902).

\section{Referate.}

I. I. Analytische Chemie.

Wm. H.Easton. Die elektrolytische Reduktion der Salpetersäure in Metallnitraten zu Ammoniak. (J. Am. Chem. Soc. 25, 1042. Oktober [6./7. 1903].)

Nach Vortmann (Berl. Berichte 23, 2798) läßt sich Salpetersäure quantitativ bestimmen, indem man ihre Lösung in Gegenwart von Kupfersulfat elektrolysiert und das gebildete Anmoniak wie üblich bestimmt. Der Verf. hat nun für dieses Verfahren die günstigsten Bedingungen angegeben. 0,5 $\mathrm{g}$ Kalisalpeter und $0,5 \mathrm{~g}$ Kupfersulfat $\left(\mathrm{CuSO}_{4}\right)$ werden zu $150 \mathrm{ccm}$ gelöst, man fügt $30 \mathrm{ccm}$ Schwefelsäure $(1,062)$ hinzu und elektro- 